\section{Antiretroviral therapy containing raltegravir to prevent mother-to-child transmission of HIV in infected pregnant women}

\author{
Diego M. Cecchini, Marina G. Martinez, \\ Laura M. Morganti, \\ Claudia G. Rodriguez \\ Hospital General de Agudos "Cosme \\ Argerich", Buenos Aires, Argentina
}

\begin{abstract}
We conducted a retrospective study in a general hospital in Buenos Aires, Argentina (2009-2015) aimed at evaluating outcomes in HIV-infected pregnant women (HIPW), who were prescribed raltegravir (RAL)containing antiretroviral therapy (ART). A total of 239 HIPW were enrolled in our study; among them 31 received RAL $(12.9 \%)$ at different clinical stages: i) intensification (INS): addition of RAL to current ART because of detectable antepartum viral load, 13 (41.9\%); ii) late presenter (LP): standard ART + RAL as fourth drug, 15 (48.4\%); iii) treatment of resistant-HIV: 3 (9.7\%). Median gestational age at RAL initiation was 34 weeks and median exposure was 30 days. In INS-group, median viral load (VL) decrease was $1.48 \log _{10}$. In LPgroup, median VL decline was $2.15 \log _{10}$ No clinical adverse events or maternal intolerance attributable to RAL were observed. Elective cesarean section was done in $51.7 \%$; mild elevation of transaminases was observed in $35 \%$ of neonates. No vertical transmission was documented.
\end{abstract}

\section{Introduction}

Use of antiretroviral drugs to prevent mother-to-child transmission (MTCT) of HIV represents one of the greatest achievements of human therapeutics. Since the successful introduction of zidovudine (AZT) as a monotherapy for the prevention of MTCT by the Pediatric AIDS Clinical Trails Group 076 trial, the prophylactic approach has evolved to the universal lifelong triple antiretroviral therapy (ART) of pregnant women in both resources rich and limited settings. Several therapeutic approaches have been evaluated to address the feasibility of preventing MTCT in resource-constrained settings most heavily affected by the epidemic. ${ }^{1}$

MTCT remains high $(>4 \%)$ in Argentina and several countries of Latin
America, including Peru, Colombia, Mexico and Paraguay, among others. Despite massive public health efforts, perinatal HIV transmission occurs often among women who present late in pregnancy, those with a high viral load due to antiretroviral drug resistance issues, non-adherence to prescribed ART, or late entry into HIV care. $^{2-4}$

Raltegravir (RAL) is an HIV-1 integrase strand transfer inhibitor (INSTI) that leads to potent viral suppression while maintaining a favorable adverse effect profile and minimal drug interactions. Its effectiveness to rapidly control HIV viral load has been demonstrated in patients with drug resistance as well as in the antiretroviralnaive population. Although there is lack of information from clinical trials regarding the use of RAL in pregnancy, there is increasing anecdotal evidence of its efficacy to rapidly reduce maternal viral load when used as a part of ART regimens late in pregnancy, with few maternal side effects and no detrimental effects on the fetus. RAL is classified as Food and Drug Administration Pregnancy Category C. The drug was neither mutagenic nor clastogenic in a series of in vitro and animal screening tests. $^{5-9}$ According to the interim Antiretroviral Pregnancy report, sufficient numbers of first trimester exposures to this drug have been monitored to detect at least a two-fold increase in risk of overall birth defects. No such increases have been detected to date. ${ }^{10}$ Recently, RAL has been included as a preferred agent in pregnancy according to the U.S. Department of Health and Human Services (DHHS) guidelines. ${ }^{11}$

In order to increase local information regarding the use of RAL in our population of HIV-infected pregnant women we underwent a retrospective study in a general hospital in Buenos Aires, Argentina during a seven year-period (2009-2015). We aimed to evaluate: i) trends in RAL prescription in HIV-infected pregnant women in our institution; ii) maternal and neonatal outcomes after RAL exposure. RAL was prescribed in this population at the standard adult dose.

\section{Materials and Methods}

During the period of analysis, a total of 239 HIV-infected pregnant women were assisted at our hospital. Of them, 31 received RAL-containing ART (12.9\%). Prescription of RAL in this population increased over time as follows: $8 / 130$ $(6.15 \%)$ in period 2009-2012 vs. 23/109 in 2013-2015 (21\%) [P<0.001, Odds Ratio (OR): 4, 95\% Confidence Interval (CI): 1.79.5].
Correspondence: Diego Martin Cecchini, Infectious Diseases Unit, Hospital Cosme Argerich, Almirante Brown 240, Buenos Aires 1155AHD, Argentina.

Tel.: +54.11.4121.0828 - Fax: +54.11.4307.5952

E-mail: diegocec@gmail.com

Key words: Pregnancy; HIV; raltegravir; antiretroviral therapy.

Contributions: DMC, conception of the work, data collection, data analysis and interpretation, drafting the article, final approval of the version to be published; MGM, data collection, data analysis and interpretation, drafting the article, final approval of the version to be published; LMM, data collection, critical revision of the article, final approval of the version to be published; CGR, data analysis and interpretation, critical revision of the article, final approval of the version to be published.

Conflict of interest: the authors declare no potential conflict of interest.

Conference presentation: data in this paper were presented in part at 21st International AIDS Conference (AIDS 2016) Durban, South Africa, July 18-22 2016 (abstract THPEC233).

Received for publication: 11 November 2016. Revision received: 13 March 2017

Accepted for publication: 17 March 2017.

This work is licensed under a Creative Commons Attribution-NonCommercial 4.0 International License (CC BY-NC 4.0).

(C) Copyright D.M. Cecchini et al., 2017 Licensee PAGEPress, Italy

Infectious Disease Reports 2017; 9:7017 doi:10.4081/idr.2017.7017

The clinical scenarios for RAL prescription were the following: i) intensification (INS, defined as addition of RAL to current ART because of detectable antepartum viral load), 13 patients (41.9\%); ii) late presenter (LP) to care (first contact with health system at $>30$ week of gestational age); those patients were prescribed standard ART according to national guidelines + RAL as fourth drug: 15 patients (48.4\%); iii) treatment of drug-resistant-HIV prior to conception: 3 patients $(9.7 \%)$.

The median (interquartile range) of age, baseline viral load and CD4 T-cell count were: 23 years (19-32); 6840 copies/mL

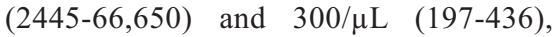
respectively. Most of the patients acquired HIV sexually, the rest perinatally. In INSgroup median viral load prior to RAL addition was 1134 copies/mL (275-29875). 
Median gestational age at RAL initiation was 34 (29-36) weeks and median exposure was 30 days.

\section{Results}

In INS-group median viral load decrease was $1.48 \log _{10}(0.98-1.77)$ and $70 \%$ had peripartum viral load $<50$ copies/mL. In LP-group, the median viral load decline was $2.15 \log _{10}(1.40-2.85)$; $45.5 \%$ and $100 \%$ had peripartum viral load $<50$ and $<400$ copies/mL, respectively. A summary of these two clinical scenarios is described in Table 1. No clinical adverse events or maternal intolerance attributable to RAL were observed, but one patient had moderate increase of transaminases.

Considering obstetric outcomes, one patient had a stillbirth; three patients had threatened preterm labor and one oligohydramnios. Three patients had premature rupture of membranes. Elective cesarean section was done in $51.7 \%$ of women and $18.5 \%$ of births were preterm. The neonatal prophylaxis prescribed was AZT in $57.1 \%$ while the rest received, combined prophylaxis (AZT + lamivudine or AZT + lamivudine + nevirapine). Most of the newborns were male $(76 \%) ; 14 \%$ had low birth weight adjusted by gestational age. Two had a ventricular septal defect (interventricular communication). Mild elevation of transaminases was observed in $35 \%$ of neonates and resolved without clinical repercussion; $14.8 \%$ of newborns required phototherapy due to hyperbilirubinemia. Three mother-child binomium were lost to follow up after delivery. No vertical transmission was documented (all infants had a negative PCR in the first week, $70 \%$ at $>2$ months and $23 \%$ had negative 18-month ELISA).

\section{Discussion}

According to recent statistics, Argentina remains far from achieving the World Health Organization goal of less than 2\% MTCT expected for non-breastfeeding populations. According to the Ministry of Health, overall MTCT rate for period 20132014 was $4.8 \%$, being $5.3 \%$ in Buenos Aires city and reaching the highest levels in the northern provinces of the country ( $>8 \%$ ). According to recent analysis of the AIDS Program of the Buenos Aires city`s Ministry of Health, inadequate obstetric control, late entry to care (and, in consequence, delayed antiretroviral therapy initiation), acute HIV infection and non-adherence to follow-up medical appointments during pregnancy were mayor determinants of perinatal transmission within the city. ${ }^{3,4}$ In this context, development of novel strategies are needed for the management of these complex clinical situations. Our mother-child cohort reflects those clinical scenarios and provides additional information to the increasing burden of evidence regard- ing the efficacy and safety of the use of RAL in pregnancy. In consequence, several aspects should be highlighted. First, rapid viral load decay was documented in LP patients (a median viral load decline was $2.15 \log _{10}$ with a median of exposure of 30 days), providing the opportunity of achieving viral loads $<400$ copies/mL by the end of pregnancy in all cases. Considering that $65-75 \%$ of HIV perinatal transmission in formula-fed populations occurs in the intrapartum period, this strategy provided a valuable opportunity for prevention of transmission during labor and delivery. ${ }^{1,8}$ In our cohort, RAL was added as fourth drug to the standard of care ART for pregnant women according to our national guidelines (two nucleosides plus a boosted protease inhibitor). ${ }^{12}$ Considering recent inclusion of RAL as a favorite drug for initiating ART during pregnancy according to recent DHHS guidelines, information regarding the viral decay with use of RAL as third drug would be crucial in order to better define the optimal therapy for LP women. ${ }^{11,13}$

Non-adherence to antiretroviral therapy and medical follow up are reflected in the INS group, in which RAL was added due to detectable viremia in late pregnancy. In this case, detectable viral load was attributable to non-adherence to antiretroviral drugs rather to virologic failure due to resistance to current therapy. This situation provided the opportunity of adding RAL to the ongoing therapy (while strengthening adherence

Table 1. Clinical characteristics and virological outcomes in HIV-infected pregnant women that received raltegravir (RAL) as part of antiretroviral therapy (ART) for prevention of perinatal transmission in two clinical scenarios. Values are numbers (median, interquartile range) unless otherwise stated.

\begin{tabular}{|c|c|c|}
\hline & Late presenter to care* $(n=15)$ & Intensification** $(n=13)$ \\
\hline Age (years) & $32(24-37.5)$ & $20(18.5-25.5)$ \\
\hline Race & Hispanic (100\%) & Hispanic (100\%) \\
\hline Mode of transmission & Sexual (100\%) & Perinatal (61.5\%), Sexual (38.5\%) \\
\hline Baseline viral load (copies/mL) & $12,217(3881-40,310)$ & $4639(1104-99,826)$ \\
\hline CD4 T cell count (cell/uL) & $372(180-578)$ & $274(170-428)$ \\
\hline Gestational age at RAL initiation (weeks) & $34(33-36)$ & $33(29-37)$ \\
\hline Pre-RAL viral load & Same as baseline & $1134(275-29,875)$ \\
\hline Median exposure & $30(7-30)$ & $25.5(7-43)$ \\
\hline Accompanying ART & $\begin{array}{c}\text { 3TC-AZT-LPV/r (73.5\%) } \\
\text { FTC-TDF-LPV/r (13.3\%) } \\
\text { 3TC-TDF-LPV/r }(6.6 \%) \\
\text { 3TC-TDF-ATV/r }(6.6 \%)\end{array}$ & $\begin{array}{c}\text { FTC-TDF-ATV/r (38.5\%) } \\
\text { 3TC-AZT-LPV/r (23\%) } \\
\text { 3TC-ABC-ATV/r (7.6\%) } \\
\text { 3TC-TDF-ATV/r (7.6\%) } \\
\text { 3TC-TDF-AZT-DRV/r (7.6\%) } \\
\text { FTC-TDF-DRV/r (7.6\%) } \\
\text { 3TC-TDF-LPV/r (7.6\%) }\end{array}$ \\
\hline ART toxicity/intolerance & Hepatotoxicity, $6.6 \%$ & None \\
\hline Median viral load decay $(\log 10)$ & $2.15(1.4-2.8)$ & $1.48(0.98-1.77)$ \\
\hline$\%$ of patients with viral load, $<400$ prior to delivery & 100 & 80 \\
\hline \% of patients with viral load, $<50$ copies/mL prior to delivery & 45.5 & 70 \\
\hline
\end{tabular}

$\%$ of patients with viral load, $<50$ copies/mL prior to delivery

*First contact with health system at $>30$ week of gestational age; **addition of RAL to ongoing antiretroviral therapy because of detectable antepartum viral load. 
strategies) in order to provide peripartum viral suppression. In this group, global viral load decay was modest compared to LP group; however pre-RAL viral load values were in general low as the patients were already on ART. Most of the patients achieved complete virological suppression prior to delivery, which can be attributed to combined effect of RAL (plus the ongoing antiretroviral therapy), improvement of adherence, or both. ${ }^{8}$

None of the patients complained of tolerance issues and no clinical adverse events were reported. One patient had asymptomatic increase of transaminases, as described in a prior publication of this cohort. ${ }^{14}$ This provides support to the increasing burden of information regarding the safety of RAL in pregnancy, as described in a recent review. ${ }^{13}$ However, baseline liver transaminases should be performed in women before starting RAL and routine monitoring should be considered. ${ }^{15}$ Of note, despite achieving virological suppression in a considerable percentage of patients, elective cesarean section was indicated in $51.7 \%$ of women and combined neonatal prophylaxis prescribed in $42.9 \%$, a higher rate than expected considering overall good virological outcomes. This situation reflects a logistical constrain, as in most cases the final pregnancy viral load was taken very few days prior to expected delivery date and results showing virologic suppression were not available for allowing a vaginal delivery. This same criteria applies for selecting combined neonatal prophylaxis instead of zidovudine alone prophylaxis.

Despite the clinically complex scenarios described in our cohort, no perinatal transmission was observed in this high risk population and no major adverse events were observed in neonates. The mild elevation of transaminases observed in $35 \%$ neonates was not attributed to RAL exposure and an interim analysis showed no differences with neonates not exposed to RAL in utero (Martinez et al., unpublished data). Further research is needed to better define impact of maternal ART on neonatal hepatotoxicity.

Major limitation of this publication is the lack of control group of pregnant women without RAL, with whom to compare viral load kinetics and achievement of virological suppression. In a recent publication, Rahangdale et al compared, in a retrospective multicentric study, the time to a clinically relevant reduction in HIV RNA in pregnant women using INSTI-containing and non-INSTI-containing antiretroviral therapy (mostly RAL). Among 90 women with a baseline HIV RNA permitting 1-log reduction, the median time to $1-\log$ RNA reduction was 8 days in the INSTI group vs. 35 days in the non-INSTI group. However, in a subgroup of 39 women with first and last RNA measurements 14 days apart this difference was reduced: the median time to 1-log reduction was 7 days in the INSTI group vs. 11 days in the non-INSTI group. ${ }^{16}$ Considering this information, clinical trials comparing antiretroviral therapies with and without RAL (and other INSTIs) are urgently needed in order to better define the optimal strategy in complex clinical scenarios as reflected in our study.

\section{Conclusions}

As far as we know, our study constitutes the biggest single center cohort of motherchild binomium exposed to RAL during pregnancy to date in Latin America and contributes to the increasing body of evidence supporting the use of this drug in pregnancy. Taking into account the high levels of MTCT in our country, inclusion of RAL in the ART of high risk-pregnant women should be strongly considered. Larger multicentric studies and clinical trials should be warranted in order to definitely establish the role of RAL for preventing MTCT.

\section{References}

1. Luzuriaga K, Mofenson LM. Challenges in the elimination of pediatric HIV-1 Infection. N Engl J Med 2016;374:761-70.

2. Pan American Health Organization. Strategy and plan of action for elimination of mother-to-child transmission of HIV and congenital syphilis in Latin America and the Caribbean: Regional Monitoring Strategy. 3. ed. Washington, D.C.: PAHO, 2013.

3. Ministerio de Salud. Boletín sobre el VIH-SIDA e ITS en la Argentina, 2015. Available from: http://www.msal.gob.ar/sida/index.php/ publicaciones/boletines-sobre-vih-sida. Accessed on 23 December 2016.

4. Coordinación SIDA Ministerio de salud. Situación epidemiológica del VIH-SIDA en la ciudad de Buenos Aires Dicembre 2014.

5. Blonk MI, Colbers APH, HidalgoTenorio C, et al. Raltegravir in HIV-1infected pregnant women: pharmacokinetics, safety, and efficacy. Clin Infect Dis 2015;61:809-16.

6. Watts DH, Stek A, Best BM, et al. Raltegravir pharmacokinetics during pregnancy for the IMPAACT 1026s study team. J Acquir Immune Defic Syndr 2014;67:375-81.

7. Nóbrega I, Travassos AG, Haguihara T, et al. Use of Raltegravir in late-presenting HIV-infected pregnant women. AIDS Res Hum Retroviruses 2013;29:145154.

8. Boucoiran I, Tulloch K, Pick N, et al. A case series of third-trimester raltegravir initiation: impact on maternal HIV-1 viral load and obstetrical outcomes. Can J Infect Dis Med Microbiol 2015;26: 145-50.

9. Croci L, Trezzi M, Allegri MP, et al. Pharmacokinetic and safety of raltegravir in pregnancy. Eur J Clin Pharmacol 2012;68:1231-2.

10. Antiretroviral Pregnancy Registry Steering Committee. Antiretroviral Pregnancy Registry International Interim Report for 1 January 1989 through 31 July 2016. Available from: http://www.APRegistry.com. Accessed on 23 December 2016.

11. Panel on Treatment of HIV-Infected Pregnant Women and Prevention of Perinatal Transmission. Recommendations for use of antiretroviral drugs in pregnant HIV-1-infected women for maternal health and interventions to reduce perinatal HIV transmission in the United States. Available from: http://aidsinfo.nih.gov/contentfiles/lvguidelines/PerinatalGL.pdf. Accessed on 23 December 2016.

12. Sociedad Argentina de Infectología. Consenso argentino de terapia antirretroviral 2014-2015. Available from: http://sadi.org.ar/publicaciones-de-lascomisiones/item/233-consenso-argentino-tarv-2014-draft. Accessed on 23 December 2016.

13. Maliakkal A, Tseng A, Walmsley SL. Review of the Efficacy, Safety, and Pharmacokinetics of Raltegravir in Pregnancy. J Acquir Immune Defic Syndr 2016;72:153-61.

14. Cecchini D, Martinez M, Morganti L, et al. [Use of raltegravir in pregnant HIV1 infected women: experience in different clinical scenarios]. Enferm Infecc Microbiol Clin 2014;32:616-8 [Article in Spanish].

15. Renet S, Closon A, Brochet MS, et al. Increase in transaminase levels following the use of raltegravir in a woman with a high HIV viral load at 35 weeks of pregnancy. J Obstet Gynaecol Canada 2013; 2013;35:68-72.

16. Rahangdale L, Cates J, Potter J, et al. Integrase inhibitors in late pregnancy and rapid HIV viral load reduction. Am J Obstet Gynecol 2016; 214:385.e1-7. 